\title{
A!
}

This is an electronic reprint of the original article.

This reprint may differ from the original in pagination and typographic detail.

Goetz, J.; Deppe, F.; Fedorov, K. G.; Eder, P.; Fischer, M.; Pogorzalek, S.; Xie, E.; Marx, A.; Gross, R.

\section{Parity-Engineered Light-Matter Interaction}

\section{Published in:}

Physical Review Letters

DOI:

10.1103/PhysRevLett.121.060503

Published: 07/08/2018

\section{Document Version}

Publisher's PDF, also known as Version of record

Please cite the original version:

Goetz, J., Deppe, F., Fedorov, K. G., Eder, P., Fischer, M., Pogorzalek, S., Xie, E., Marx, A., \& Gross, R. (2018). Parity-Engineered Light-Matter Interaction. Physical Review Letters, 121(6), [060503].

https://doi.org/10.1103/PhysRevLett.121.060503

This material is protected by copyright and other intellectual property rights, and duplication or sale of all or part of any of the repository collections is not permitted, except that material may be duplicated by you for your research use or educational purposes in electronic or print form. You must obtain permission for any other use. Electronic or print copies may not be offered, whether for sale or otherwise to anyone who is not an authorised user. 


\title{
Parity-Engineered Light-Matter Interaction
}

\author{
J. Goetz,$^{1,2, *}$ F. Deppe, ${ }^{1,2,3}$ K. G. Fedorov, ${ }^{1,2}$ P. Eder, ${ }^{1,2,3}$ M. Fischer, ${ }^{1,2,3}$ S. Pogorzalek, ${ }^{1,2}$ E. Xie, ${ }^{1,2,3}$ \\ A. Marx, ${ }^{1}$ and R. Gross ${ }^{1,2,3, \dagger}$ \\ ${ }^{1}$ Walther-Meißner-Institut, Bayerische Akademie der Wissenschaften, 85748 Garching, Germany \\ ${ }^{2}$ Physik-Department, Technische Universität München, 85748 Garching, Germany \\ ${ }^{3}$ Nanosystems Initiative Munich (NIM), Schellingstraße 4, 80799 München, Germany
}

(Received 15 March 2018; revised manuscript received 15 May 2018; published 7 August 2018)

\begin{abstract}
The concept of parity describes the inversion symmetry of a system and is of fundamental relevance in the standard model, quantum information processing, and field theory. In quantum electrodynamics, parity is conserved and large field gradients are required to engineer the parity of the light-matter interaction operator. In this work, we engineer a potassiumlike artificial atom represented by a specifically designed superconducting flux qubit. We control the wave function parity of the artificial atom with an effective orbital momentum provided by a resonator. By irradiating the artificial atom with spatially shaped microwave fields, we select the interaction parity in situ. In this way, we observe dipole and quadrupole selection rules for single state transitions and induce transparency via longitudinal coupling. Our work advances the design of tunable artificial multilevel atoms to a new level, which is particularly promising with respect to quantum chemistry simulations with near-term superconducting circuits.
\end{abstract}

DOI: 10.1103/PhysRevLett.121.060503

Parity and its underlying symmetries play an elementary role in pioneering theories such as $C P$ violation [1], the Higgs formalism [2], and quantum phase transitions [3]. Parity measurements are essential in quantum information processing [4-6], field theory [7,8], and light-matter interaction [9-11]. In the latter, the application of highfrequency electromagnetic radiation in resonance with a desired state transition reveals the internal structure of matter. Depending on both symmetry and strength of the probe field, various multipole moments can be activated [12] and the corresponding selection rules (SRs) become apparent [13]. In atomic systems, their natural properties limit systematic studies of SRs and often the interaction is dominated by the odd-parity dipole operator. Because of the small atomic length scale, even-parity quadrupole interactions are mostly relevant for large field gradients. Here, we therefore use a specific superconducting artificial atom $[14,15]$, which is suitable for future experiments in quantum simulation and quantum chemistry $[16,17]$. In comparison to previous studies [10,18-25], our approach aims for the simulation of systems including orbital momentum by choosing a qubit architecture, where a single loop introduces a magnetic dipole moment and a gradiometer loop gives rise to a magnetic quadrupole moment. With those spatial degrees of freedom, we create an artificial potassium-like atom by introducing an effective orbital momentum provided by the wave function parity of a superconducting resonator. A carefully tailored pair of antennas additionally allows us to tune the parity of the light-matter interaction operator. Adjusting drive amplitude and phase, we can invert the rf magnetic field within the dimensions of the atom, which is a unique property in quantum optics. Changing the interaction parity is possible because the dipole moment provides longitudinal coupling [26] (even interaction parity) and the quadrupole moment transversal coupling (odd interaction parity) [9]. With this implementation we induce transparency $[23,27]$ even when the potential of the artificial atom on its own does not exhibit a well-defined symmetry. Using the dispersively coupled resonator, we activate dipole forbidden transitions via sideband transitions [28]. The ability to address dipolar and quadrupolar transitions in a single artificial atom together with a more complex level structure is of potential use in future quantum simulations of chemical compounds and quantum annealing.

As shown in Fig. 1(a), we couple a tunable-gap gradiometric flux qubit $[14,15]$ galvanically to the fundamental current mode of a half-wavelength coplanar waveguide resonator operating at $\omega_{\mathrm{r}} / 2 \pi \simeq 3.9 \mathrm{GHz}$ [29]. In the first part of this work, we use the resonator only for readout purposes and in the second part, we additionally use the resonator to probe symmetry effects beyond the twolevel atom approximation. The qubit itself consists of a gradiometric aluminum loop patterned on a high-resistivity silicon substrate [Fig. 1(b)]. The center conductor is interrupted by two Josephson junctions and a dc SQUID where the latter provides an effective Josephson junction with a tunable critical current. To avoid interface losses [41], the sample is fabricated from a single $\mathrm{Al} / \mathrm{AlO}_{\mathrm{x}} / \mathrm{Al}$ trilayer structure using the shadow evaporation technique [42]. We mount the sample to the $25 \mathrm{mK}$ base temperature stage of a custom-made dilution refrigerator. The resonator 
(a)

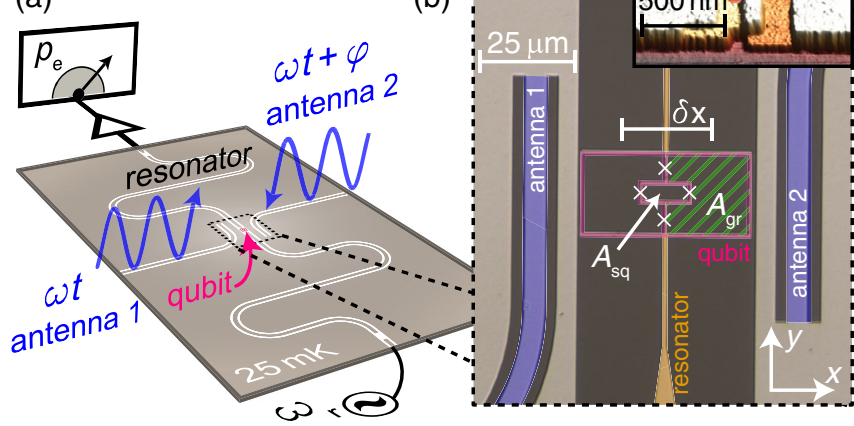

(c)

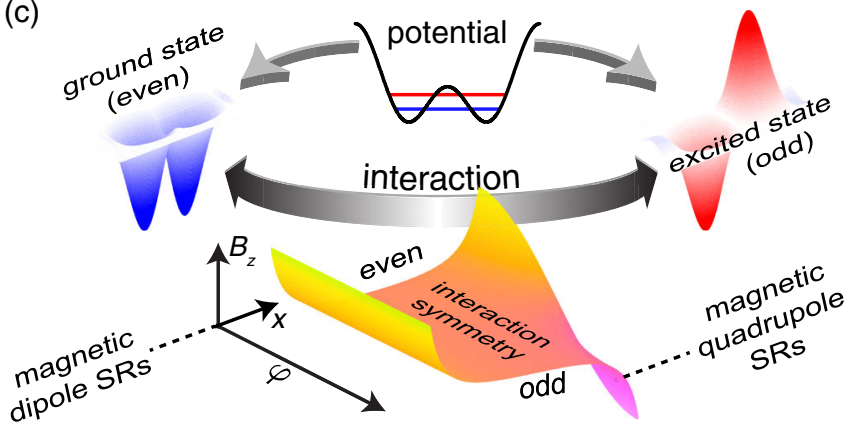

FIG. 1. (a) Chip layout and detection scheme. (b) False-colored micrograph of the qubit architecture. Crosses indicate Josephson junctions as the one shown in the atomic force micrograph (inset). The SQUID is placed on the symmetry axis of the qubit and the center point of the two gradiometer loops (green shaded area). (c) The symmetric double well potential for flux qubits results in two eigenstates with opposite parity (blue and red wave functions). The interaction is defined by the symmetry of a drive field that can be even, odd, or without a specific symmetry, controlled by the relative phase $\varphi$ between two frequency-degenerate microwave drives.

with Hamiltonian $\hat{\mathcal{H}}_{\mathrm{r}}=\hbar \omega_{\mathrm{r}} \hat{a}^{\dagger} \hat{a}$ is characterized by its external coupling rate $\kappa_{\mathrm{x}} / 2 \pi \simeq 2.47 \mathrm{MHz}$ and internal loss rate $\kappa_{\mathrm{i}} / 2 \pi \simeq 70 \mathrm{kHz}$. The qubit excited state probability $p_{e}$ and the coherence times $T_{1} \simeq 2.6$ and $T_{2} \simeq 0.1 \mu \mathrm{s}$ are obtained with a dispersive readout scheme [43]. The qubit-resonator coupling strength $g / 2 \pi \simeq 41 \mathrm{MHz}$ is predominantly transversal and is described by a light-matter interaction term $\hat{\mathcal{H}}_{\mathrm{qr}}=\hbar\left(g_{\mathrm{t}} \cos \theta \hat{\sigma}_{z}-g_{\mathrm{t}} \sin \theta \hat{\sigma}_{x}\right)\left(\hat{a}^{\dagger}+\hat{a}\right)$. We trap one flux quantum in the outer qubit loop to generate a double well potential [Fig. 1(c)]. In terms of the odd- and even-parity Pauli operators $\hat{\sigma}_{x}$ and $\hat{\sigma}_{z}$, the resulting Hamiltonian reads $\hat{\mathcal{H}}_{\mathrm{q}}^{\prime}=\left(\Delta \hat{\sigma}_{x}+\varepsilon \hat{\sigma}_{z}\right) / 2$. We set the tunnel coupling between the wells to $\Delta / \hbar \simeq 2 \pi \times$ $8.2 \mathrm{GHz}$ and vary the energy bias $\varepsilon$ with a pair of onchip antennas placed symmetrically with respect to the qubit. The relevant $z$ component of the magnetic field irradiated by the antennas contains dc and ac components, i.e., $B_{z}(x, y)=B_{z}^{\mathrm{dc}}(x, y)+B_{z}^{\mathrm{ac}}(x, y) \cos \omega t$, where $\omega / 2 \pi$ is the drive frequency. Depending on the relative phase $\varphi$ between the two drive fields, we generate either a symmetric field configuration, $B_{z, \mathrm{sq}} \equiv \Phi_{\mathrm{sq}} / \mathcal{A}_{\mathrm{sq}}$ and $\delta B_{z, \mathrm{gr}} / \delta x=0$, or antisymmetric field gradients, $\delta B_{z, \mathrm{gr}} / \delta x \equiv$ $\left(2 \Phi_{\mathrm{gr}} / \mathcal{A}_{\mathrm{gr}}\right) / \delta x$ and $B_{z, \mathrm{gr}}=0$. The fluxes $\Phi_{\mathrm{sq}}$ and $\Phi_{\mathrm{gr}}$ are the integrals of $B_{z}(x, y)$ over the areas $\mathcal{A}_{\mathrm{sq}}$ and $\mathcal{A}_{\mathrm{gr}}$ of the SQUID and a single gradiometer loop, respectively [see Fig. 1(b)]. For arbitrary $\varphi$, the total field $B_{z, \text { tot }}=B_{z, \mathrm{sq}}+$ $\delta B_{z, \mathrm{gr}} / \delta x$ is a superposition of symmetric and antisymmetric contributions.

Because of the elaborate sample geometry, the two terms of $B_{z \text {,tot }}$ couple to different Pauli operators in the Hamiltonian $\hat{\mathcal{H}}=p B_{z, \mathrm{sq}} \hat{\sigma}_{z}+Q\left(\delta B_{z, \mathrm{gr}} / \delta x\right) \hat{\sigma}_{x}$ [29]. Here, the SQUID dipole moment $p$ and the gradiometer quadrupole moment $Q$ define the longitudinal and transversal coupling strengths $\Omega_{\ell}(\varphi)=p B_{z, \mathrm{sq}}^{\text {ac }}(\varphi) / \hbar$ and $\Omega_{\mathrm{t}}(\varphi)=$ $Q\left[\delta B_{z, \text { gr }}^{\text {ac }}(\varphi) / \delta x\right] / \hbar$, respectively. Hence, the qubit couples longitudinally to symmetric fields $(\varphi \in\{0,2 \pi, \ldots\} \rightarrow$ $\left.\Omega_{\mathrm{t}}=0\right)$ and transversally to antisymmetric fields $\left(\varphi \in\{\pi, 3 \pi, \ldots\} \rightarrow \Omega_{\ell}=0\right)$. In the qubit energy eigenbasis, where $\hat{\mathcal{H}}_{\mathrm{q}}=\hbar \omega_{\mathrm{q}} \hat{\sigma}_{z} / 2 \equiv \sqrt{\Delta^{2}+\varepsilon^{2}} \hat{\sigma}_{z} / 2$ and $\theta=$ $\tan ^{-1}(\Delta / \varepsilon)$ is the Bloch angle controlled by $B_{z}^{\mathrm{dc}}$, the interaction Hamiltonian reads

$$
\begin{aligned}
\hat{\mathcal{H}}_{\text {int }}= & \hbar \cos (\omega t)\left[\left(\Omega_{\ell}(\varphi) \cos \theta-\Omega_{\mathrm{t}}(\varphi) \sin \theta\right) \hat{\sigma}_{x}\right. \\
& \left.+\left(\Omega_{\mathrm{t}}(\varphi) \cos \theta+\Omega_{\ell}(\varphi) \sin \theta\right) \hat{\sigma}_{z}\right] / 2 .
\end{aligned}
$$

In addition to the field symmetry characterized by $\Omega_{\ell}$ and $\Omega_{\mathrm{t}}$ as described above, the parity of $\hat{\mathcal{H}}_{\text {int }}$ depends on the symmetry of the qubit wave functions. The latter is governed by the qubit parity operator $\hat{\Pi}_{\mathrm{q}}=-\hat{\sigma}_{z}$, which results in an odd parity for pure $\hat{\sigma}_{x}$ interaction, an even parity for pure $\hat{\sigma}_{z}$ interaction, and no well-defined parity when both terms are present [10]. Even interaction parity results in forbidden transitions (transparency) because the commutator $\left[\hat{\Pi}_{\mathrm{q}}, \hat{\sigma}_{z}\right]=0$. Then, $\hat{\sigma}_{z}$ is a constant of motion, $\imath \hbar \partial \hat{\sigma}_{z} / \partial t=0$, and the qubit remains in the ground state with a modulated qubit gap $\Delta$. Since, in contrast to Ref. [10], we activate $\hat{\sigma}_{x}$ with an antisymmetric drive field at $\theta=\pi / 2$, the most natural interpretation of our system is given in terms of magnetic SRs.

In a first experiment, we investigate the two-antenna control of the drive symmetry required to observe magnetic SRs. We operate at the point of optimal phase coherence, $\theta=\pi / 2$, where the qubit potential is symmetric and initial state $|\mathrm{g}\rangle$ and final $|\mathrm{e}\rangle$ state have opposite parity [9], $\Pi_{\mathrm{i}}=-\Pi_{\mathrm{f}}$. In Fig. 2(a), we plot the qubit excited state probability $p_{\mathrm{e}}=\left(\left\langle\hat{\sigma}_{z}\right\rangle+1\right) / 2$ for different spatial distributions controlled by the relative phase $\varphi$. The resonant drive $\left(\omega=\omega_{\mathrm{q}}\right)$ is kept at constant power and is split symmetrically into the two antenna lines [29]. We observe oscillations between $p_{\mathrm{e}} \simeq 0$ and $p_{\mathrm{e}} \simeq 0.5$ occurring when $\varphi$ assumes integer multiples of $\pi$. For these values, also the drive has a well-defined parity which leads to the formation of magnetic SRs. As long as $\varphi$ is not set to an integer multiple of $\pi$, we shape a drive field without specific 


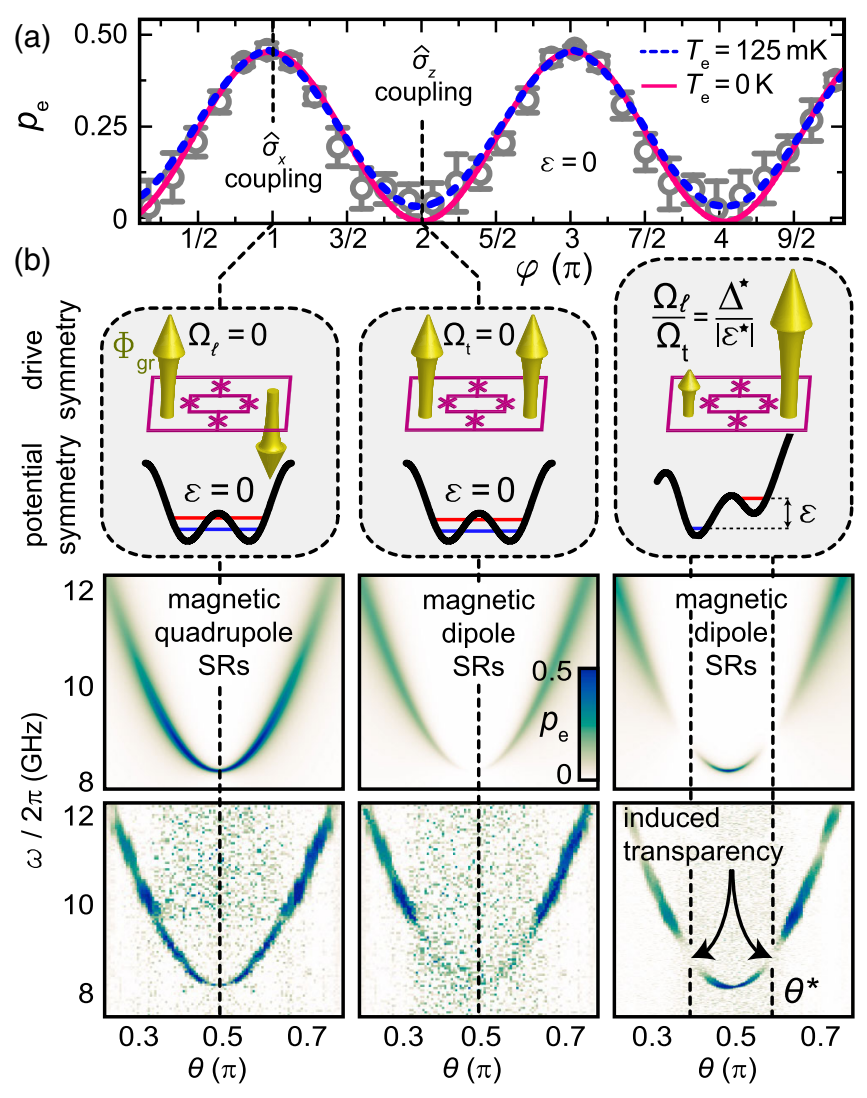

FIG. 2. (a) Qubit excited state probability $p_{e}$ plotted versus

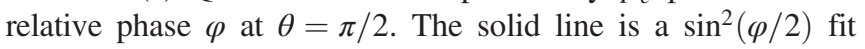
assuming the qubit to be at zero temperature, the dashed line takes a finite qubit temperature into account and the error bars are of statistical nature. (b) Top panel: Symmetry of the drive field amplitude and the qubit potential. Lower panel: Simulated (top row) and measured (bottom row) excited state probability $p_{e}$ plotted versus the Bloch angle $\theta$ and the drive frequency $\omega / 2 \pi$. At $\theta^{\star}$ we observe longitudinal coupling-induced transparency. We attribute the scatter between $0.3 \pi \lesssim \theta \lesssim 0.6 \pi$ to variations in the signal-to-noise ratio of our measurement setup. The reduced signal strength at $\theta \simeq 0.7$ in the bottom right panel is not predicted by our model and could be due to a stray microwave mode or a two-level defect coupled to the qubit.

symmetry $\left(\Omega_{\mathrm{t}}, \Omega_{\ell} \neq 0\right)$. Then, there are no SRs and $p_{\mathrm{e}}$ follows qualitatively the expected $\sin ^{2}(\varphi / 2)$ dependence. There is a small stray excitation probability $p_{\mathrm{e}}^{\text {str }} \simeq 0.05=$ $\exp \left[-\hbar \omega_{\mathrm{q}} /\left(k_{B} T_{e}\right)\right]$ for pure $\hat{\sigma}_{z}$ interaction, which can be linked to an effective temperature $T_{e}$. We attribute this effect to a constant thermal contribution in the transversal drive, $\hbar \Omega_{\mathrm{t}} \mapsto \hbar \Omega_{\mathrm{t}}(\varphi)+p_{e}^{\text {str }} \hbar \omega_{q}$. Using this ansatz, we find quantitative agreement between theory and experiment for $T_{e} \simeq 125 \mathrm{mK}$. Similar values are found in other experiments on superconducting circuits [43-45].

In the next step, we demonstrate an enhanced level of control by investigating SRs for a tilted qubit potential, $\theta \neq \pi / 2$. To this end, we measure the averaged excited state probability $p_{e}$ as a function of drive frequency $\omega$ and potential tilt, which is controlled by $\theta$. In the cases of purely symmetric and antisymmetric drives, which are shown in the left and middle column of Fig. 2(b), the broken symmetry of the qubit potential leads to the absence of SRs [10]. Clearly, the transition is allowed for both symmetric and antisymmetric drive fields away from the optimal point. Our specific qubit and antenna geometry allow us to restore the broken symmetry and observe rigorous SRs also for a tilted qubit potential. To this end, we sweep $\theta$ for a certain ratio $\Omega_{\ell} / \Omega_{\mathrm{t}} \simeq 30$ and observe a strong decrease in $p_{e}$ for $\theta^{\star} \simeq 0.4 \pi$ and $\theta^{\star} \simeq 0.6 \pi$ as shown in the right column of Fig. 2(b). At these points, the ratio $\Omega_{\ell} / \Omega_{\mathrm{t}}$ is equal to the asymmetry parameter $\tan \theta^{\star} \equiv$ $\Delta^{\star} /\left|\varepsilon^{\star}\right|$ of the qubit potential. In other words, we exploit that the presence of SRs is determined by the symmetry properties of the interaction operator $\hat{\mathcal{H}}_{\text {int }}$, rather than by those of the qubit alone. The vanishing transition matrix element at $\theta^{\star}$ is known as longitudinal coupling-induced transparency [27]. It can be understood from a formal point of view, because the $\hat{\sigma}_{x}$ term in the Hamiltonian of Eq. (1) vanishes when $\Omega_{\ell} / \Omega_{\mathrm{t}}=\Delta^{\star} /\left|\varepsilon^{\star}\right|$. In this case, one can rotate into a basis where both qubit potential and drive field are symmetric such that the interaction Hamiltonian becomes $\hat{\mathcal{H}}_{\text {int }}^{\star}=\Omega_{\mathrm{t}} \hat{\sigma}_{z} / \cos \theta^{\star}$.

So far, our discussion has been restricted to an artificial two-level atom, where the qubit states $|\mathrm{g}\rangle$ and $|e\rangle$ behave similar to the $1 s$ and $2 p$ state of a hydrogen atom, respectively, and transparency due to longitudinal coupling could be induced. To observe also the opposite phenomenon, the activation of transversally forbidden transitions between states of equal parity, we engineer a more complex artificial atom using the dispersively coupled resonator. In the parity operator for the resonator, [6] $\hat{\Pi}_{\mathrm{r}}=e^{i \pi n}$, the eigenvalues $n$ of the photon number operator $\hat{n}$ play the role of the quantum number of an orbital momentum in natural atoms. As shown in Fig. 3(a), we now exploit the parity [8] $\hat{\Pi}_{\mathrm{q}} \hat{\Pi}_{\mathrm{r}}=-\hat{\sigma}_{z} e^{l \pi n}$ of the combined qubit-resonator system and map the state $|\mathrm{g}, n\rangle$ to the even $s$ orbital. Similarly, when the qubit is in the excited state, the resonator states $|n+1\rangle,|n\rangle$, and $|n-1\rangle$ give rise to artificial even $s$, odd $p$, and even $d$ orbitals, respectively. We note that, although the qubit parity enters into the details of our mapping, it is essentially the resonator, which acts as an artificial orbital momentum. In this way, the qubit-resonator system qualitatively mimics the energy levels and parity properties of a potassium atom with electron configuration $[\mathrm{Ar}] 4 s^{1}$ [see Fig. 3(a)]. The corresponding electric SRs are precisely reproduced if we realize the symmetric (antisymmetric) electric drive as a physical antisymmetric (symmetric) magnetic field. In order to avoid confusion with our previous discussion of magnetic SRs, we prefer to present the experimental results in the more universal language of transversal and longitudinal coupling. We first investigate the effect of SRs between states of equal parity by probing the red and blue sideband transitions, $|\mathrm{g}, n\rangle \mapsto|e, n \pm 1\rangle$ 


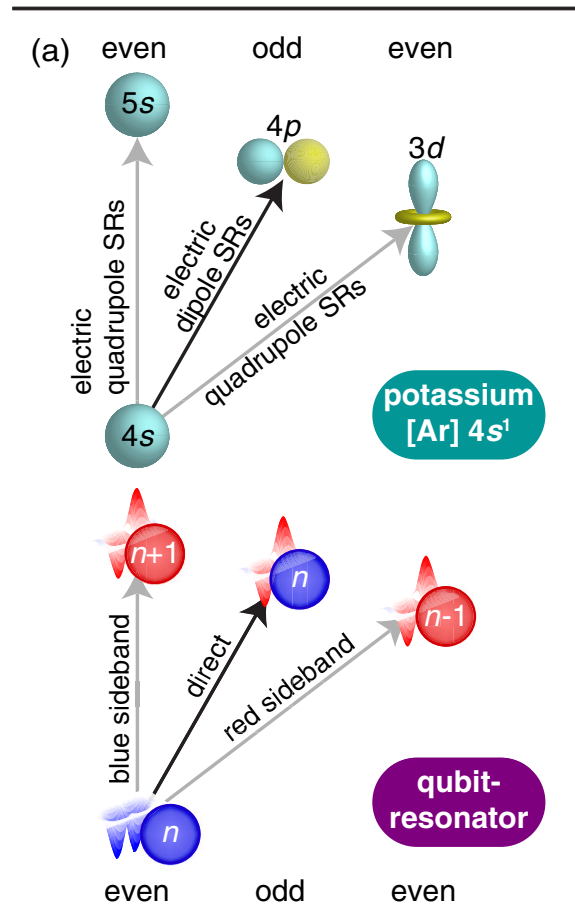

(b)

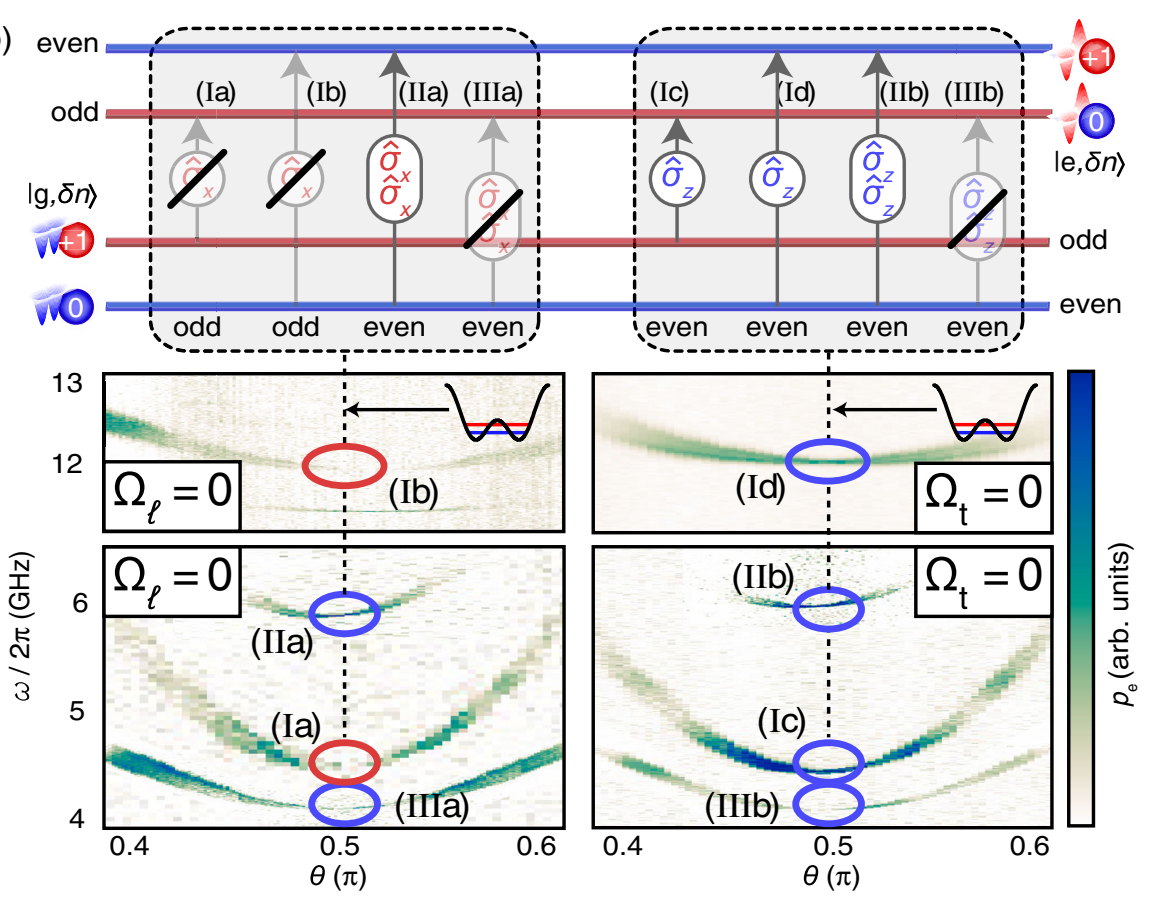

FIG. 3. (a) Energy level diagram for ground and first three excited states for a potassium atom (top) and the qubit-resonator system (bottom). The atom follows electric SRs, which have a close analogy to sideband transitions in the qubit-resonator system. (b) Top panel: Level scheme and corresponding parity of composite qubit-resonator states for multiphoton transitions $|\mathrm{g}, \delta n\rangle \mapsto|e, \pm 1\rangle$. Crossed out arrows denote forbidden transitions. Lower panel: Qubit excited state probability $p_{e}$ plotted versus Bloch angle $\theta$ and drive frequency $\omega / 2 \pi$. The circled areas show the red and blue sidebands (Ia-Id), the two-photon transition of the blue sideband (IIa and IIb), and the direct two-photon transition (IIIa and IIIb) for a symmetric qubit potential. Because of different power levels required to drive the transitions, the data are an overlay of different measurements. The vertical shift between the cases IIa and IIb is caused by small flux jumps between the measurements.

[cases Ia-Id in Fig. 3(b)]. To this end, we probe the resonator response at frequency $\omega_{r}$ while simultaneously driving the sidebands at frequencies $\omega=\omega_{q} \pm \omega_{r}$. The photon required for the absorption process of the red sideband transition, $|\mathrm{g}, n\rangle \mapsto|e, n-1\rangle$, is provided by the readout tone, which contains approximately $\langle n\rangle \simeq 30$ photons. Without longitudinal coupling $\left(\Omega_{\ell}=0\right)$, resonant transitions between states of equal parity are forbidden and both sidebands vanish at $\theta=\pi / 2$. Changing the relative phase $\varphi$ to apply a purely longitudinal drive $\left(\Omega_{t}=0\right)$, these transitions become allowed and we observe a strong increase in $p_{e}$ at $\theta=\pi / 2$. Interestingly, the transition is always allowed for the twophoton blue sideband [case (IIa) and case (IIb)] and always forbidden for the two-photon qubit excitation [case (IIIa) and case (IIIb) in Fig. 3(b)]. The reason for this behavior is that applying an odd or an even operator twice always produces an even-parity operator [28]. Despite the more complex scenario, all transitions are allowed when the symmetry of the qubit potential or the interaction is not purely transversal or purely longitudinal. We note that higher-order sideband transitions can be neglected in our experiments because they are detuned strongly in frequency and typically require a much higher driving power to be activated.
In conclusion, we have established a superconducting architecture allowing for full in situ control over the parity of light-matter interaction and the participating quantum states. We have selectively addressed the magnetic dipole and quadrupole moment of our artificial atom and verified the corresponding SRs. Furthermore, we show that a dispersively coupled resonator acts as an artificial orbital momentum and enables the simulation of multilevel artificial atoms. Even though the potassium level structure could, in principle, be obtained using other qubit types, we find that the precise shaping of interaction symmetries works particularly well with the gradiometric tunable-gap flux qubit. This central result will be essential for future simulations of quantum chemistry $[16,17,46]$. Especially regarding near-term quantum devices with a relatively small number of quantum circuits, the prospect of obtaining a higher complexity from replacing qubits with more realistic artificial multilevel atoms is very appealing. In the system discussed here, even spin-orbit coupling could be realized by adding resonators with tunable qubitresonator coupling strengths. More generally speaking, the coexistence of longitudinal and transversal coupling is important to reach the nonstoquastic regime in adiabatic quantum computing [47,48] and quantum annealing [49-52]. Based on our results on highly tunable qubit-drive 
coupling, the controlled coexistence of transversal and longitudinal interactions between two gradiometric tunable-gap qubits appears feasible by designing their magnetic field distribution, by coupling the qubit to quantum circuits providing symmetric and antisymmetric modes [53,54], or using a parametric drive. In addition, for increasing coupling strengths, longitudinal coupling terms may lead to new experimental studies of the symmetries in the quantum Rabi model [7,55] and help to obtain the quantum Fisher information for probabilistic parameters in time-dependent Hamiltonians [56]. The range of possible applications can be even further extended by considering longitudinal qubitresonator coupling [57], quantum state engineering [58-60], and the simulation of relativistic physics [61].

We thank E. Solano and D. Braak for fruitful discussions. We acknowledge financial support from the German Research Foundation through SFB 631 and FE 1564/1-1, the doctorate program ExQM of the Elite Network of Bavaria, and the International Max Planck Research School "Quantum Science and Technology." The authors declare no competing financial interests.

*jan.goetz@wmi.badw.de

Present address: QCD Labs, Department of Applied Physics, Aalto University, Aalto, Finland.

†rudolf.gross@wmi.badw.de

[1] C. S. Wu, E. Ambler, R. W. Hayward, D. D. Hoppes, and R. P. Hudson, Phys. Rev. 105, 1413 (1957).

[2] P. W. Higgs, Phys. Rev. Lett. 13, 508 (1964).

[3] A. Trenkwalder, G. Spagnolli, G. Semeghini, S. Coop, M. Landini, P. Castilho, L. Pezze, G. Modugno, M. Inguscio, A. Smerzi, and M. Fattori, Nat. Phys. 12, 826 (2016).

[4] D. Riste, C. C. Bultink, M. J. Tiggelman, R. N. Schouten, K. W. Lehnert, and L. DiCarlo, Nat. Commun. 4, 1913 (2013).

[5] A. Córcoles, E. Magesan, S. J. Srinivasan, A. W. Cross, M. Steffen, J. M. Gambetta, and J. M. Chow, Nat. Commun. 6, 6979 (2015).

[6] N. Ofek, A. Petrenko, R. Heeres, P. Reinhold, Z. Leghtas, B. Vlastakis, Y. Liu, L. Frunzio, S. M. Girvin, L. Jiang, M. Mirrahimi, M. H. Devoret, and R. J. Schoelkopf, Nature (London) 536, 441 (2016).

[7] S. Felicetti, T. Douce, G. Romero, P. Milman, and E. Solano, Sci. Rep. 5, 11818 (2015).

[8] R. Birrittella, K. Cheng, and C. C. Gerry, Opt. Commun. 354, 286 (2015).

[9] Y. X. Liu, J. Q. You, L. F. Wei, C. P. Sun, and F. Nori, Phys. Rev. Lett. 95, 087001 (2005).

[10] F. Deppe, M. Mariantoni, E. P. Menzel, A. Marx, S. Saito, K. Kakuyanagi, H. Tanaka, T. Meno, K. Semba, H. Takayanagi, E. Solano, and R. Gross, Nat. Phys. 4, 686 (2008).

[11] A. Kou, W. C. Smith, U. Vool, R. T. Brierley, H. Meier, L. Frunzio, S. M. Girvin, L. I. Glazman, and M. H. Devoret, Phys. Rev. X 7, 031037 (2017).
[12] P. K. Jain, D. Ghosh, R. Baer, E. Rabani, and A. P. Alivisatos, Proc. Natl. Acad. Sci. U.S.A. 109, 8016 (2012).

[13] R. J. Rafac, B. C. Young, J. A. Beall, W. M. Itano, D. J. Wineland, and J. C. Bergquist, Phys. Rev. Lett. 85, 2462 (2000).

[14] F. G. Paauw, A. Fedorov, C. J. P. M. Harmans, and J. E. Mooij, Phys. Rev. Lett. 102, 090501 (2009).

[15] M. J. Schwarz, J. Goetz, Z. Jiang, T. Niemczyk, F. Deppe, A. Marx, and R. Gross, New J. Phys. 15, 045001 (2013).

[16] S. Mostame, P. Rebentrost, A. Eisfeld, A. J. Kerman, D. I. Tsomokos, and A. Aspuru-Guzik, New J. Phys. 14, 105013 (2012).

[17] S. Mostame, J. Huh, C. Kreisbeck, A. J. Kerman, T. Fujita, A. Eisfeld, and A. Aspuru-Guzik, Quantum Inf. Process. 16, 44 (2017).

[18] S. J. Srinivasan, A. J. Hoffman, J. M. Gambetta, and A. A. Houck, Phys. Rev. Lett. 106, 083601 (2011).

[19] J. Q. You and F. Nori, Nature (London) 474, 589 (2011).

[20] P. C. de Groot, J. Lisenfeld, R. N. Schouten, S. Ashhab, A. Lupascu, C. J. P. M. Harmans, and J. E. Mooij, Nat. Phys. 6, 763 (2010).

[21] N. Didier, J. Bourassa, and A. Blais, Phys. Rev. Lett. 115, 203601 (2015).

[22] P. Forn-Díaz, G. Romero, C. J. P. M. Harmans, E. Solano, and J. E. Mooij, Sci. Rep. 6, 26720 (2016).

[23] Y. Wu, L.-P. Yang, Y. Zheng, H. Deng, Z. Yan, Y. Zhao, K. Huang, W. J. Munro, K. Nemoto, D.-N. Zheng, C. P. Sun, Y.-x. Liu, X. Zhu, and L. Lu, arXiv:1605.06747.

[24] B. Royer, A. L. Grimsmo, N. Didier, and A. Blais, Quantum 1, 11 (2017).

[25] U. Vool, A. Kou, W. C. Smith, N. E. Frattini, K. Serniak, P. Reinhold, I. M. Pop, S. Shankar, L. Frunzio, S. M. Girvin, and M. H. Devoret, Phys. Rev. Applied 9, 054046 (2018).

[26] P.-M. Billangeon, J. S. Tsai, and Y. Nakamura, Phys. Rev. B 91, 094517 (2015).

[27] Y. Liu, C.-X. Yang, H.-C. Sun, and X.-B. Wang, New J. Phys. 16, 015031 (2014).

[28] A. Blais, J. Gambetta, A. Wallraff, D. I. Schuster, S. M. Girvin, M. H. Devoret, and R. J. Schoelkopf, Phys. Rev. A 75, 032329 (2007).

[29] See Supplemental Material at http://link.aps.org/ supplemental/10.1103/PhysRevLett.121.060503 for experimental techniques and theoretical methods, which includes Refs. [30-40].

[30] A. Abragam, The Principles of Nuclear Magnetism, 32 (Oxford University Press, New York, 1961).

[31] T. P. Orlando, J. E. Mooij, L. Tian, C. H. van der Wal, L. S. Levitov, S. Lloyd, and J. J. Mazo, Phys. Rev. B 60, 15398 (1999).

[32] D. I. Schuster, A. Wallraff, A. Blais, L. Frunzio, R.-S. Huang, J. Majer, S. M. Girvin, and R. J. Schoelkopf, Phys. Rev. Lett. 94, 123602 (2005).

[33] R. E. Raab and O.L. De Lange, Multipole Theory in Electromagnetism: Classical, Quantum, and Symmetry Aspects, with Applications (Oxford University Press on Demand, Oxford, 2005), Vol. 128.

[34] C. Cohen-Tannoudji, B. Diu, and F. Laloe, Quantum Mechanics, 2 (John Wiley \& Sons, New York, 2006).

[35] J. Gambetta, A. Blais, M. Boissonneault, A. A. Houck, D. I. Schuster, and S. M. Girvin, Phys. Rev. A 77, 012112 (2008). 
[36] M. Boissonneault, J. M. Gambetta, and A. Blais, Phys. Rev. A 79, 013819 (2009).

[37] T. Niemczyk, F. Deppe, H. Hübl, E. P. Menzel, F. Hocke, M. J. Schwarz, J. J. Garcia-Ripoll, D. Zueco, T. Hümmer, E. Solano, A. Marx, and R. Gross, Nat. Phys. 6, 772 (2010).

[38] T. Niemczyk, F. Deppe, E. P. Menzel, M. J. Schwarz, H. Huebl, F. Hocke, M. Häberlein, M. Danner, E. Hoffmann, A. Baust, E. Solano, J. J. Garcia-Ripoll, A. Marx, and R. Gross, arXiv:1107.0810.

[39] M. Kasperczyk, S. Person, D. Ananias, L. D. Carlos, and L. Novotny, Phys. Rev. Lett. 114, 163903 (2015).

[40] J. Goetz, F. Deppe, P. Eder, M. Fischer, M. Müting, J. P. Martínez, S. Pogorzalek, F. Wulschner, E. Xie, K. G. Fedorov, A. Marx, and R. Gross, Quant. Sci. Tech. 2, 025002 (2017).

[41] J. Goetz, F. Deppe, M. Haeberlein, F. Wulschner, C. W. Zollitsch, S. Meier, M. Fischer, P. Eder, E. Xie, K. G. Fedorov, E. P. Menzel, A. Marx, and R. Gross, J. Appl. Phys. 119, 015304 (2016).

[42] G. J. Dolan, Appl. Phys. Lett. 31, 337 (1977).

[43] J. Goetz, S. Pogorzalek, F. Deppe, K. G. Fedorov, P. Eder, M. Fischer, F. Wulschner, E. Xie, A. Marx, and R. Gross, Phys. Rev. Lett. 118, 103602 (2017).

[44] F. Yan, S. Gustavsson, A. Kamal, J. Birenbaum, A. P. Sears, D. Hover, T. J. Gudmundsen, D. Rosenberg, G. Samach, S. Weber, J. L. Yoder, T. P. Orlando, J. Clarke, A. J. Kerman, and W. D. Oliver, Nat. Commun. 7, 12964 (2016).

[45] K. Y. Tan, M. Partanen, R. E. Lake, J. Govenius, S. Masuda, and M. Möttönen, Nat. Commun. 8, 15189 (2017).

[46] A. Potočnik, A. Bargerbos, F. A. Y. N. Schröder, S. A. Khan, M. C. Collodo, S. Gasparinetti, Y. Salathé, C. Creatore, C. Eichler, H. E. Türeci, A. W. Chin, and A. Wallraff, Nat. Commun. 9, 904 (2018).
[47] L. Hormozi, E. W. Brown, G. Carleo, and M. Troyer, Phys. Rev. B 95, 184416 (2017).

[48] T. Albash and D. A. Lidar, Rev. Mod. Phys. 90, 015002 (2018).

[49] J. Brooke, D. Bitko, F. T. Rosenbaum, and G. Aeppli, Science 284, 779 (1999).

[50] D. Aharonov, W. van Dam, J. Kempe, Z. Landau, S. Lloyd, and O. Regev, SIAM J. Comput. 37, 166 (2007).

[51] T. Lanting et al., Phys. Rev. X 4, 021041 (2014).

[52] W. Vinci and D. A. Lidar, npj Quantum Inf. 3, 38 (2017).

[53] A. Baust, E. Hoffmann, M. Haeberlein, M. J. Schwarz, P. Eder, J. Goetz, F. Wulschner, E. Xie, L. Zhong, F. Quijandría, B. Peropadre, D. Zueco, J.-J. García Ripoll, E. Solano, K. Fedorov, E. P. Menzel, F. Deppe, A. Marx, and R. Gross, Phys. Rev. B 91, 014515 (2015).

[54] A. Baust, E. Hoffmann, M. Haeberlein, M. J. Schwarz, P. Eder, J. Goetz, F. Wulschner, E. Xie, L. Zhong, F. Quijandría, D. Zueco, J.-J. Garcia Ripoll, L. García-Álvarez, G. Romero, E. Solano, K. G. Fedorov, E. P. Menzel, F. Deppe, A. Marx, and R. Gross, Phys. Rev. B 93, 214501 (2016).

[55] D. Braak, Phys. Rev. Lett. 107, 100401 (2011).

[56] S. Pang and A. N. Jordan, Nat. Commun. 8, 14695 (2017).

[57] S. Richer and D. DiVincenzo, Phys. Rev. B 93, 134501 (2016).

[58] D. Porras and J. J. García-Ripoll, Phys. Rev. Lett. 108, 043602 (2012).

[59] F. Quijandría, D. Porras, J. J. García-Ripoll, and D. Zueco, Phys. Rev. Lett. 111, 073602 (2013).

[60] Y.-J. Zhao, Y.-L. Liu, Y.-x. Liu, and F. Nori, Phys. Rev. A 91, 053820 (2015).

[61] M. del Rey, D. Porras, and E. Martin-Martinez, Phys. Rev. A 85, 022511 (2012). 